Claremont Colleges

Scholarship@ Claremont

All HMC Faculty Publications and Research

HMC Faculty Scholarship

3-1-2005

\title{
Combinatorial Proofs of Fermat's, Lucas's, and Wilson's Theorems
}

Peter G. Anderson

Rochester Institute of Technology

Arthur T. Benjamin

Harvey Mudd College

Jeremy A. Rouse

University of Wisconsin - Madison

\section{Recommended Citation}

Anderson, Peter G., Arthur T. Benjamin and Jeremy A. Rouse. "Combinatorial Proofs of Fermat's, Lucas's, and Wilson's Theorems." The American Mathematical Monthly, Vol 112, No. 3, pp. 266-268, March 2005.

This Article is brought to you for free and open access by the HMC Faculty Scholarship at Scholarship @ Claremont. It has been accepted for inclusion in All HMC Faculty Publications and Research by an authorized administrator of Scholarship @ Claremont. For more information, please contact scholarship@cuc.claremont.edu. 


\section{MAA}

Combinatorial Proofs of Fermat's, Lucas's, and Wilson's Theorems

Author(s): Peter G. Anderson, Arthur T. Benjamin and Jeremy A. Rouse

Source: The American Mathematical Monthly, Vol. 112, No. 3 (Mar., 2005), pp. 266-268

Published by: Mathematical Association of America

Stable URL: http://www.jstor.org/stable/30037444

Accessed: 10/06/2013 18:39

Your use of the JSTOR archive indicates your acceptance of the Terms \& Conditions of Use, available at

http://www.jstor.org/page/info/about/policies/terms.jsp

JSTOR is a not-for-profit service that helps scholars, researchers, and students discover, use, and build upon a wide range of content in a trusted digital archive. We use information technology and tools to increase productivity and facilitate new forms of scholarship. For more information about JSTOR, please contact support@ jstor.org. 
It is clear that $L_{(P)}$ is a linear operator from $\mathcal{H}_{k}$ to $\mathcal{H}_{k}$. Moreover, we claim that $L_{(P)}$ is self-adjoint and positive. In fact, for any $u$ in $\mathcal{H}_{m+k}$ and $v$ in $\mathcal{H}_{k}$ we infer from (2) that

$$
\langle P(\partial) u, v\rangle_{k}=\langle u, \bar{P} v\rangle_{k+m} .
$$

It follows that for $Q$ and $R$ in $\mathcal{H}_{k}$

$$
\begin{aligned}
\left\langle L_{(P)}[Q], R\right\rangle_{k} & =\langle P(\partial)(P Q), R\rangle_{k}=\langle\bar{P} Q, \bar{P} R\rangle_{k+m} \\
& =\langle Q, P(\partial)(\bar{P} R)\rangle_{k}=\left\langle Q, L_{(P)}[R]\right\rangle_{k},
\end{aligned}
$$

which means $L_{(P)}$ is self-adjoint.

Analogously, by (4) we have

$$
\left\langle L_{(P)}[Q], Q\right\rangle_{k}=\langle\bar{P} Q, \bar{P} Q\rangle_{k+m}=\|\bar{P} Q\|_{k+m}^{2} \geq\|P\|_{m}^{2}\|Q\|_{k}^{2},
$$

showing that $L_{(P)}$ is positive.

Therefore, we see that all eigenvalues of $L_{(P)}$ are positive and, on the basis of (5), that $\|P\|_{m}^{2}$ furnishes a lower bound for them. Furthermore, equality holds in (4) if and only if either $P \equiv 0$ or $\|P\|_{m}^{2}$ is the smallest eigenvalue of $L_{(P)}$ and $Q$ is an eigenvector corresponding to it (unless $Q \equiv 0$ ). A particular case in which equality holds in (4) occurs when $P=P(y)$ belongs to $\mathcal{H}_{m}$ and $Q=Q(z)$ to $\mathcal{H}_{k}$, where $y \in \mathbb{R}^{p}, z \in \mathbb{R}^{q}$, and $\mathbb{R}^{n}=\mathbb{R}^{p} \times \mathbb{R}^{q}$.

Added in proof. Professor Luo Xuebo, who was one of his coauthor's Ph.D. supervisors, died in March 2004. Zhu-Jun Zheng expresses his deep respect for and everlasting memory of his deceased colleague and mentor.

Institute of Applied Mathematics, Northwestern Polytechnical University, Xi'an, 710072, P. R. China.

Institue of Mathematics, Henan University, Kaifeng, 475001, P. R. China.

zhengzj@henu.edu.cn

\section{Combinatorial Proofs of Fermat's, Lucas's, and Wilson's Theorems}

\section{Peter G. Anderson, Arthur T. Benjamin, and Jeremy A. Rouse}

In this note, we observe that many classical theorems from number theory are simple consequences of the following combinatorial lemma:

Lemma 1. Let $S$ be a finite set, let $p$ be prime, and suppose $f: S \rightarrow S$ has the property that $f^{p}(x)=x$ for any $x$ in $S$, where $f^{p}$ is the p-fold composition of $f$. Then $|S| \equiv|F|(\bmod p)$, where $F$ is the set of fixed points of $f$.

Proof. The set $S$ is the disjoint union of sets of the form $\left\{x, f(x), \ldots, f^{p-1}(x)\right\}$. Since $p$ is prime, each set has either size one or size $p$.

The Lucas numbers 2, 1, 3, 4, 7, 11, 18, 29, 47, ., named in honor of Edouard Lucas (1842-1891), are defined by $L_{0}=2, L_{1}=1$, and $L_{n}=L_{n-1}+L_{n-2}$ for $n \geq 2$. 
It is easy to show that, for $n \geq 1, L_{n}$ counts the ways to create a bracelet of length $n$ using beads of length one or two, where bracelets that differ by a rotation or a reflection are still considered distinct. For example, there are four bracelets of length three. (Such a bracelet can have three beads of length one, or it can have a bead of length two and a bead of length one, where the bead of length one can be in position one, two, or three.) Let $f$ act on bracelets of prime length $p$ by rotating each bead clockwise one unit. Clearly $f^{p}$ leaves any bracelet unchanged. Since $f$ has just one fixed point (when all beads have length one), we conclude that $L_{p} \equiv 1(\bmod p)$ for each prime $p$.

More generally, for nonnegative integers $a$ and $b$ the Lucas sequence (of the second kind) is defined, as in [4], by $V_{0}=2, V_{1}=a$, and $V_{n}=a V_{n-1}+b V_{n-2}$ for $n \geq 2$. Again, it is easy to show [1] that $V_{n}$ with $n \geq 1$ counts colored bracelets of length $n$, where there are $a$ color choices for beads of length one and $b$ color choices for beads of length two. By the same argument as earlier, with the exception of those bracelets consisting of length one beads all of the same color, when $p$ is prime every bracelet can be rotated to create $p$ distinct bracelets. Thus

$$
V_{p} \equiv a \quad(\bmod p)
$$

for each prime $p$. In the special case where $b=0$, it is clear that $V_{p}=a^{p}$. Consequently, we have Fermat's Theorem: if $p$ is a prime, then

$$
a^{p} \equiv a \quad(\bmod p)
$$

This combinatorial proof of Fermat's theorem was originally given in [2].

Next, consider colored bracelets of length $p k$, where $p$ is prime. If we rotate the beads $k$ units at a time, then there are exactly $V_{k}$ fixed points, obtained by taking any colored bracelet of length $k$ and "replicating" it $p$ times. Our lemma concludes that for $p$ prime

$$
V_{p k} \equiv V_{k} \quad(\bmod p)
$$

In particular, $V_{p^{e}} \equiv V_{p^{e-1}}$ when $e \geq 1$. Consequently, for $p$ prime and $e$ nonnegative,

$$
V_{p^{e}} \equiv a \quad(\bmod p)
$$

Now consider the set $S$ of permutations of $\{0,1, \ldots, p-1\}$ with exactly one cycle; thus, $|S|=(p-1)$ !. Define $f: S \rightarrow S$ by

$$
f\left(\left(a_{0}, a_{1}, \ldots, a_{p-1}\right)\right)=\left(1+a_{0}, 1+a_{1}, \ldots, 1+a_{p-1}\right),
$$

where addition is done modulo $p$. For each $\pi$ in $S, f^{p}(\pi)=\pi$. For $a$ satisfying $1 \leq$ $a \leq p-1$ those permutations of the form $\pi_{a}=(0, a, 2 a, 3 a, \ldots,(p-1) a)$ (with multiplication done modulo $p$ ) are fixed points of $f$ since $f\left(\pi_{a}\right)$ remains an "arithmetic progression." Conversely, if $\pi$ is a fixed point of $f$ and $\pi(0)=a$, then $\pi=$ $f^{a}(\pi)$ must send $a$ to $2 a$ and, in general, $\pi=f^{k a}(\pi)$ sends $k a$ to $(k+1) a$. Thus $\pi=\pi_{a}$, and $f$ has exactly $p-1$ fixed points. This establishes Wilson's Theorem: if $p$ is a prime, then

$$
(p-1) ! \equiv(p-1) \quad(\bmod p)
$$

The same approach can be applied to the set $S$ of $k$-element subsets of

$$
\{0,1, \ldots, p-1\} \text {. }
$$


Define $f: S \rightarrow S$ by $f\left(\left\{a_{1}, a_{2}, \ldots, a_{k}\right\}\right)=\left\{1+a_{1}, 1+a_{2}, \ldots, 1+a_{k}\right\}$, where again addition is done modulo $p$. When $1 \leq k \leq p-1$ there are no fixed points of $f$. Consequently, for $p$ prime and $k$ satisfying $1 \leq k \leq p-1$,

$$
\left(\begin{array}{l}
p \\
k
\end{array}\right) \equiv 0 \quad(\bmod p) .
$$

We conclude with Lucas's Theorem: For $p$ prime, let $n$ and $k$ have base $p$ notation $n=\sum_{i \geq 0} b_{i} p^{i}$ and $k=\sum_{i \geq 0} c_{i} p^{i}$, where $0 \leq b_{i}, c_{i}<p$. Then

$$
\left(\begin{array}{l}
n \\
k
\end{array}\right) \equiv \prod_{i \geq 0}\left(\begin{array}{l}
b_{i} \\
c_{i}
\end{array}\right) \quad(\bmod p) .
$$

Proof. It suffices to show that $\left(\begin{array}{l}p n+r \\ p k+s\end{array}\right) \equiv\left(\begin{array}{l}n \\ k\end{array}\right)\left(\begin{array}{l}r \\ s\end{array}\right)(\bmod p)$ when $0 \leq r, s<p$, and then proceed inductively. Let $S$ denote the set of ordered pairs $(A, v)$, where $A$ is a binary $p \times n$ matrix and $v$ is a binary $r \times 1$ vector such that among the $p n+r$ entries of $A$ and $v$ exactly $p k+s$ are equal to one. Hence $|S|=\left(\begin{array}{c}p n+r \\ p k+s\end{array}\right)$. Let $Q$ denote the $p \times p$ permutation matrix with nonzero entries $q_{1 p}=1$ and $q_{i, i-1}=1$ for $i=2,3, \ldots, p$. Thus $Q A$ has the same rows as $A$, each shifted "down" by one row.

Define $f: S \rightarrow S$ by $f((A, v))=(Q A, v)$. Then $f^{p}((A, v))=\left(Q^{p} A, v\right)=$ $(A, v)$. There are $\left(\begin{array}{l}n \\ k\end{array}\right)\left(\begin{array}{l}r \\ s\end{array}\right)$ fixed points of $f$, consisting of those pairs $(A, v)$ such that the first row of $A$ contains exactly $k$ ones, the other rows of $A$ are the same as the first row, and $v$ contains exactly $s$ ones in its $r$ positions. Note that if $s>r$, then $\left(\begin{array}{l}r \\ s\end{array}\right)=0$. Thus, by our lemma, $\left(\begin{array}{c}p n+r \\ p k+s\end{array}\right) \equiv\left(\begin{array}{l}n \\ k\end{array}\right)\left(\begin{array}{l}r \\ s\end{array}\right)(\bmod p)$, as desired.

For another fine combinatorial proof of Lucas's theorem, see [3].

ACKNOWLEDGMENT. The authors gratefully acknowledge valuable suggestions from David Gaebler and the anonymous referee.

\section{REFERENCES}

1. A. T. Benjamin and J. J. Quinn, Proofs That Really Count, The Art of Combinatorial Proofs, Mathematical Association of America, Providence, 2003.

2. L. E. Dickson, History of the Theory of Numbers, vol. 1, Carnegie Institution of Washington, Washington, D.C., 1919.

3. N. J. Fine, Binomial coefficients modulo a prime, this MonThLy 54 (1947) 589-592.

4. P. Ribenboim, The Little Book of Big Primes, Springer-Verlag, New York, 1991.

Department of Computer Science, Rochester Institute of Technology, Rochester, NY 14623-5608 anderson@cs.rit.edu

Department of Mathematics, Harvey Mudd College, Claremont, CA 91711

benjamin@hmc.edu

Department of Mathematics, University of Wisconsin, Madison, WI 53706

rouse@math.wisc.edu 